\title{
Diagnosis and Prognosis of Scrubber Faults for Underwater Rebreathers based on Stochastic Event Models
}

\author{
Dongsik Chang and Fumin Zhang \\ School of Electrical and \\ Computer Engineering \\ Georgia Institute of Technology \\ Atlanta, Georgia 30332 \\ Email: dsfrancis3,fumin@gatech.edu
}

\author{
Michael West \\ Georgia Tech Research Institute \\ Atlanta, Georgia 30332 \\ Email: Mick.West@gtri.gatech.edu
}

\begin{abstract}
Imperfect $\mathrm{CO}_{2}$ removal mechanisms of $\mathrm{CO}_{2}$ scrubbers often lead to the existence of $\mathrm{CO}_{2}$ in gas inhaled by a diver from underwater rebreathers. This may cause $\mathrm{CO}_{2}$ related rebreather faults and subsequently would increase the risk of human injuries. We introduce a stochastic model for three $\mathrm{CO}_{2}$ related rebreather faults: $\mathrm{CO}_{2}$ bypass, scrubber exhaustion, and scrubber breakthrough. We establish the concept of $\mathrm{CO}_{2}$ channeling that describes the cause of the faults and present a $\mathrm{CO}_{2}$ channeling model based on a stochastic process driven by a Poisson counter. This helps us to investigate how $\mathrm{CO}_{2}$ flow inside the rebreather is affected by $\mathrm{CO}_{2}$ related faults. Fault diagnosis/prognosis algorithms are developed based on the stochastic model and are tested in simulation.
\end{abstract}

\section{INTRODUCTION}

Underwater breathing apparatus (UBA) are used for dive assistance. In consideration of military use or saturation diving, one of the most widely used UBA is the closed circuit rebreather (CCR) [1]. A rebreather reuses exhaled gas from divers by employing a scrubber to remove $\mathrm{CO}_{2}$ from the exhaled gas. However, $\mathrm{CO}_{2}$ removal mechanisms are usually not perfect. The phenomena of excessive $\mathrm{CO}_{2}$ in inhaled gas and the loss of $\mathrm{CO}_{2}$ absorption capability of the scrubber are considered as severe faults of the rebreather, which may lead to life threatening injuries to divers due to the intake of the excessive $\mathrm{CO}_{2}$ [2], [3], [4].

Efforts to analyze or detect possible faults of rebreathers have been made in [4], [5], [6]. Deep Life, Ltd. published research data on rebreathers, and their work including a MATLAB/SIMULINK rebreather model is accessible [4]. A computer simulation model for the chemical kinetics of $\mathrm{CO}_{2}$ absorption by a scrubber was developed in [5] to investigate the characteristics of the scrubber. This model is utilized to monitor the status of the scrubber by observing temperature changes induced by chemical reactions between $\mathrm{CO}_{2}$ and the scrubing chemicals. In addition, a fault tree was designed in [6] to identify risks of rebreather faults.

Aside from rebreather fault modeling, the physical characteristics of UBA such as gas dynamics under pressure at various locations are modeled in [7], [8], [9]. A computer simulation tool [7] analyzes the overall gas flow in the UBA. The breathing dynamics of closed-circuit UBA or CCRs are discussed in [8]. The main interest there is how to maximize performance in terms of breathing characteristics including work of breathing and peak to peak pressure. The authors of [9] extended [7], [8] by applying network theory to describe components in the breathing loop that affect gas flow.

In this paper, we focus on $\mathrm{CO}_{2}$ related faults of a rebreather and model how $\mathrm{CO}_{2}$ flow inside the rebreather is affected by $\mathrm{CO}_{2}$ related faults. The faults we are interested in include $\mathrm{CO}_{2}$ bypass, scrubber exhaustion, and scrubber breakthrough. We define a random event that a small amount of $\mathrm{CO}_{2}$ passes through the scrubber without being absorbed as $\mathrm{CO}_{2}$ channeling, which can be described by a Poisson process. Accumulated $\mathrm{CO}_{2}$ channeling will lead to the faults. The $\mathrm{CO}_{2}$ flow will be affected by the faults and can be modeled using stochastic differential equations.

Our approach on the stochastic representation of $\mathrm{CO}_{2}$ related rebreather faults is novel. The model captures the stochastic nature of faults that are difficult to detect and predict. This leads to the development of stochastic model based fault diagnosis/prognosis algorithms for $\mathrm{CO}_{2}$ related rebreahter faults without modeling the process of chemical reactions inside a scrubber which might require more computing power. Our contribution may lead to detection and prediction of scrubber faults in real time.

In the next section, we briefly explain terminologies and mechanisms of rebreathers, and in Section III, we model respiration of divers that provides the input and the output of a rebreather. In Section IV, we simplify gas dynamics of an oxygen rebreather to focus on $\mathrm{CO}_{2}$ related scrubber faults and investigate the influence of the faults on $\mathrm{CO}_{2}$ flow in the simplified rebreather by introducing a $\mathrm{CO}_{2}$ channeling model driven by a stochastic process. The description of the particle filter for the $\mathrm{CO}_{2}$ flow model is presented in Section $\mathrm{V}$ followed by the introduction to fault diagnosis and prognosis for $\mathrm{CO}_{2}$ related rebreather faults in Section VI. Simulation results are shown in Section VII and conclusions are provided 
in Section VIII.

\section{MECHANiSMS OF A REBREATHER}

As shown in Fig. 1, a typical oxygen rebreather consists of a mouth-piece, a scrubber canister, an oxygen cylinder and a pressure gauge. Air flow inside a rebreather is driven by a diver's exhalation and inhalation forming a closed breathing loop [10]. From the viewpoint of the scrubber, the portion of the breathing loop where gas flows from the diver through the mouth-piece to the scrubber is named the incoming path, and the portion of the breathing loop where gas flows out of the scrubber back to the diver is named the outgoing path. The direction of gas flow is regulated by one-way valves in the mouth-piece. When a diver inhales, the valves open the outgoing path and block the incoming path so that the gas in the incoming path cannot be breathed back into the diver. In contrast, when a diver exhales, the valves close the outgoing path and direct the exhaled gas to the incoming path to prevent it from being mixed with the gas in the outgoing path.

During the use of the rebreather, pure oxygen is injected into the breathing loop from the oxygen cylinder. This can be performed either actively at a constant rate, which must satisfy the individual diver's personal needs based on the diver's metabolism, or by manually adding oxygen using a hand-operated valve. It can also be controlled based on the difference between ambient pressure and the breathing loop pressure. The pressure of the oxygen inside the cylinder is checked with the pressure gauge.

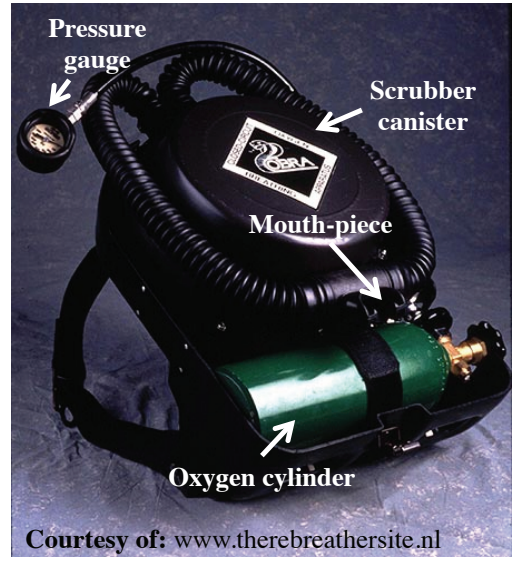

Fig. 1. A pure oxygen rebreather (Cobra).

$\mathrm{CO}_{2}$ is absorbed by the scrubber using chemicals such as sodalime stored in the canister [11], [12], [13]. The scrubber has a limited life time since the reaction of the scrubbing chemicals with $\mathrm{CO}_{2}$ is irreversible. In fact, even when sodalime is not fully consumed, $\mathrm{CO}_{2}$ may pass through the scrubber by a large amount, causing $\mathrm{CO}_{2}$ related faults. Thus, the status of the scrubber consumption should be monitored in order for divers to avoid severe injuries due to the intake of excessive $\mathrm{CO}_{2}$, i.e., hypercapnia. However, due to their stochastic nature, these faults are difficult to predict, and when they happen, divers often have little time to react. One of our goals is to understand the stochastic nature of the $\mathrm{CO}_{2}$ related faults towards better predictions.

\section{Modeling Inhalation AND Exhalation RATES}

Divers interact with rebreathers by respiration, i.e., inhalation and exhalation. A rebreather can be viewed as a system that takes exhalation as an input and produces an output for inhalation. An advanced computer simulation model of the human respiration is dealt with in [14] and its application to semi-closed circuit underwater breathing equipment is discussed in [15]. For rebreather fault simulations, a simplistic respiration model is constructed in this section in accordance with European Standard EN14143:2003 [16].

European standard EN14143:2003 [16] requires a sinusoidal waveform of breathing simulator and recommends $\mathrm{CO}_{2}$ absorption endurance test at ventilation rate $40 \mathrm{~L} / \mathrm{min}$ and at $\mathrm{CO}_{2}$ generation rate $1.6 \mathrm{~L} / \mathrm{min}$. Moreover, the breathing simulator should handle breathing frequency of $20 / \mathrm{min}$ when ventilation rate is $40 \mathrm{~L} / \mathrm{min}$. According to these recommendations, we define the ventilation rate $r$ and the frequency $f$ as $r=40[\mathrm{~L} / \mathrm{min}]$ and $f=\frac{1}{3}[\mathrm{~Hz}]$. Note that the period $T$ is just $\frac{1}{f}$ such that $T=3[s]$.

In reality, respiration rates are different from person to person even among people who have similar physical conditions. We can easily see that the respiration rate is expected to vary along with workload changes which have influence on the heart rate. Let us define $s=\frac{\mathrm{HR}}{\mathrm{HR}_{\mathrm{ref}}}$ as the heart rate ratio where $H_{\text {ref }}$ is the reference heart rate and HR is the present heart rate. Let $\xi(s)$ be a function which affects the magnitude of the respiration rate and $\chi(s)$ be a function which affects the frequency of the respiration rate. Based on the above discussion, the normalizing factor $L$, the inhalation rate $v$ and the exhalation rate $u$ with heart rate variations are

$$
\begin{aligned}
& L(s, t)=\left\{\begin{array}{ll}
\int_{\frac{n T}{\chi(s)}}^{\frac{\left(n+\frac{1}{2}\right) T}{\chi(s)}} \sin (2 \pi f \chi(s) t) d t & , t \in I_{1} \\
\int_{\frac{(n+1) T}{\chi(s)}}^{\frac{\left(n+\frac{1}{2}\right) T}{\chi(s)}} \sin (2 \pi f \chi(s) t+\pi) d t & , t \in I_{2}
\end{array},\right. \\
& v(s, t)= \begin{cases}\frac{r \xi(s)}{60 f L(s, t)} \sin (2 \pi f \chi(s) t) & , t \in I_{1}, \\
0 & , t \in I_{2}\end{cases} \\
& u(s, t)= \begin{cases}0, t \in I_{1} \\
\frac{r \xi(s)}{60 f L(s, t)} \sin (2 \pi f \chi(s) t+\pi)\end{cases}
\end{aligned}
$$

where $\xi(1)=1, \chi(1)=1, I_{1}=\left[\frac{n T}{\chi(s)}, \frac{\left(n+\frac{1}{2}\right) T}{\chi(s)}\right)$ and $I_{2}=$ $\left[\frac{\left(n+\frac{1}{2}\right) T}{\chi(s)}, \frac{(n+1) T}{\chi(s)}\right)$ for $n=\{0,1,2, \cdots\}$.

The inhalation rate and the exhalation rate are listed separately as in (2) and (3) since inhalation and exhalation do not happen at the same time. A combination of one cycle of 
inhalation and one cycle of exhalation produces a single cycle of respiration. In other words, in the case that the heart rate ratio $s$ is one and the respiration rate is $1 / 3 \mathrm{~Hz}$, then each of inhalation and exhalation takes place in every 1.5 seconds, leading to a cycle of respiration in every 3 seconds.

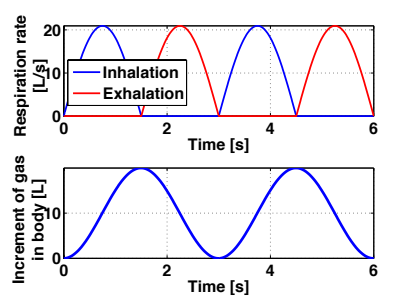

(a) Respiration rate over time with unit heart rate ratio
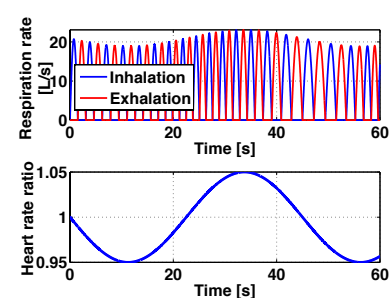

(b) Respiration rate along with heart rate change
Fig. 2. In (a), the top plot shows rate of inhalation and exhalation for each cylce, and the bottom plot shows the amount of gas ventilated through a diver. In (b), the top plot shows the variation of the respiration rate related to the heart rate ratio shown in the bottom plot.

Fig. 2 demonstrates our respiration model. In the top row, the blue and the red curves indicate inhalation and exhalation rates, respectively. The respiration rate and the amount of gas flow with no heart rate changes, i.e., $s=1$, are shown in Fig. 2(a). The inhalation and exhalation rates when the heart rate varies are demonstrated in Fig. 2(b).

\section{Modeling $\mathrm{CO}_{2}$ Flow}

In this section, we analyze how $\mathrm{CO}_{2}$ related faults affect $\mathrm{CO}_{2}$ flow based on the following assumptions which simplify gas dynamics in a rebreather: (i) when a diver breathes out, exhaled gas flows to a scrubber canister instantly, i.e., we ignore gas dynamics in the incoming path; (ii) elements of gas are uniformly distributed throughout the outgoing path and the ratio of the gas elements breathed in by a diver is identical to the ratio of the gas elements in the outgoing path; (iii) the amount of $\mathrm{CO}_{2}$ breathed in by a diver is breathed out later without loss.

We first model $\mathrm{CO}_{2}$ flow in each case of inhalation and exhalation assuming that $\mathrm{CO}_{2}$ is absorbed by an ideal $\mathrm{CO}_{2}$ scrubber. The two separate parts based on inhalation and exhalation can later be combined. We describe $\mathrm{CO}_{2}$ related faults following a stochastic approach and analyze how $\mathrm{CO}_{2}$ flow is affected by $\mathrm{CO}_{2}$ related faults using a stochastic representation of the faults.

\section{A. $\mathrm{CO}_{2}$ Flow with no Fault}

The volume of $\mathrm{CO}_{2}$ in the outgoing path will be measured using proper $\mathrm{CO}_{2}$ sensors. Let $x$ be the volume of $\mathrm{CO}_{2}$ in the outgoing path. We define the ratio of $\mathrm{CO}_{2}$ in the outgoing path $c_{1}$ as

$$
c_{1}(t)=\frac{x(t)}{V_{L}}
$$

where $V_{L}$ is the volume of the total gas in the outgoing path.
During the inhalation cycle, the volume of inhaled $\mathrm{CO}_{2}$ is determined by the inhalation rate $v$ and the ratio of $\mathrm{CO}_{2}$ in the outgoing path $c_{1}$. Let us define $y$ as the volume of $\mathrm{CO}_{2}$ removed by a scrubber. By our assumptions, gas in the incoming path does not flow to the outgoing path during inhalation, so $\mathrm{CO}_{2}$ absoprtion $y$ is zero. On the other hand, the amount of $\mathrm{CO}_{2}$ in the outgoing path $x$ will be reduced due to the inhalation by a diver. Therefore, $\mathrm{CO}_{2}$ flow during inhalation $\left(t \in I_{1}\right)$ is described as

$$
\begin{aligned}
\dot{x} & =-c_{1}(t) \cdot v(t) \\
& =-\frac{v(t)}{V_{L}} \cdot x(t) \\
\dot{y} & =0
\end{aligned}
$$

where $x(0)=0, y(0)=0$ and $I_{1}=\left[\frac{n T}{\chi(s)}, \frac{\left(n+\frac{1}{2}\right) T}{\chi(s)}\right)$ for $n=$ $\{0,1,2, \cdots\}$.

We now consider the exhalation cycle. According to [17], the volume of $\mathrm{CO}_{2}$ generated by a diver changes along with the heart rate variations. However, the variations of the $\mathrm{CO}_{2}$ generation rate, which is related to $\mathrm{O}_{2}$ metabolic consumption rate, based on workload is not easy to predict [10]. We use a simplified model based on the heart rate ratio here. We assume that the change in $\mathrm{CO}_{2}$ generation rate is proportional to the heart rate ratio $s$. Let us define the $\mathrm{CO}_{2}$ generation ratio $c_{2}$ as

$$
c_{2}(s)=a \cdot s
$$

where $a$ is a scaling factor with a typical value $a=0.04$ and $s$ is the heart rate ratio.

In the ideal cases, the scrubber absorbs all the $\mathrm{CO}_{2}$ coming into a scrubber during exhalation. In other words, no $\mathrm{CO}_{2}$ will flow to the outgoing path of a rebreather. The volume of $\mathrm{CO}_{2}$ coming into a scrubber is determined by the exhalation rate $u$ and the $\mathrm{CO}_{2}$ generation ratio $c_{2}$ Thus, $x$ and $y$ during exhalation $\left(t \in I_{2}\right)$ can be described as follows.

$$
\begin{aligned}
& \dot{x}=0 \\
& \dot{y}=c_{2}(s) \cdot u(t)
\end{aligned}
$$

where $x(0)=0, y(0)=0, I_{2}=\left[\frac{\left(n+\frac{1}{2}\right) T}{\chi(s)}, \frac{(n+1) T}{\chi(s)}\right)$ for $n=$ $\{0,1,2, \cdots\}$ and $s$ is the heart rate ratio. When $\mathrm{CO}_{2}$ related faults happen, $\mathrm{CO}_{2}$ flow will change as will be discussed in the following subsections.

\section{B. Influence of $\mathrm{CO}_{2}$ related Faults on Respiration}

Since $\mathrm{CO}_{2}$ related faults cause $\mathrm{CO}_{2}$ leaks to the outgoing path from the incoming path without being absorbed by a scrubber, the volume of $\mathrm{CO}_{2}$ in the outgoing path $x$ during exhalation is not zero anymore. Moreover, divers inhale $\mathrm{CO}_{2}$ and this affects the volume of $\mathrm{CO}_{2}$ contained in exhaled breath. Assuming that human-beings do not consume $\mathrm{CO}_{2}$, then $\mathrm{CO}_{2}$ breathed in will remain intact in exhaled gas. Let us define $\delta x$ describing the total amount of $\mathrm{CO}_{2}$ inhaled by a diver over one inhalation period as 


$$
\delta x(n)=x(n T)-x\left(\left(n+\frac{1}{2}\right) T\right)
$$

where $n=\{0,1,2, \cdots\}$. Assuming that all the $\mathrm{CO}_{2}$ inhaled by a diver is exhaled during the next exhalation cycle without loss, we define an additional exhalation rate $u_{1}$ for this portion of returned $\mathrm{CO}_{2}$ as follows.

$$
u_{1}(t, n)= \begin{cases}0 & , t \in I_{1} \\ \frac{\delta x(n)}{L} \sin (2 \pi f t+\pi) & , t \in I_{2}\end{cases}
$$

where $I_{1}=\left[\frac{n T}{\chi(s)}, \frac{\left(n+\frac{1}{2}\right) T}{\chi(s)}\right)$ and $I_{2}=\left[\frac{\left(n+\frac{1}{2}\right) T}{\chi(s)}, \frac{(n+1) T}{\chi(s)}\right)$ for $n=$ $\{0,1,2, \cdots\}$.

Considering that the returned $\mathrm{CO}_{2}$ is not involved in $\mathrm{CO}_{2}$ generation, we define the volume of $\mathrm{CO}_{2}$ in exhaled breath using $u$ and $u_{1}$ as

$$
\mathscr{U}(t, n)=\left\{\begin{array}{cc}
0 & , t \in I_{1} \\
c_{2}(s)\left(u(t)-u_{1}(t, n)\right)+u_{1}(t, n) & , t \in I_{2}
\end{array}\right.
$$

where $s$ is the heart rate ratio.

\section{C. $\mathrm{CO}_{2}$ Channeling and Stochastic Modeling}

We focus on the following $\mathrm{CO}_{2}$ related rebreather faults: $\mathrm{CO}_{2}$ bypass, scrubber exhaustion, and scrubber breakthrough. The descriptions of the faults are as follows. (i) $\mathrm{CO}_{2}$ bypass: Existence of $\mathrm{CO}_{2}$ beyond a safety level in the outgoing path of the rebreather. (ii) Scrubber exhaustion: Complete consumption of the $\mathrm{CO}_{2}$ scrubber. (iii) Scrubber breakthrough: Loss of the $\mathrm{CO}_{2}$ absorption capability of the $\mathrm{CO}_{2}$ scrubber before the depletion of the scrubber. We will see later that these faults can be rigorously defined using a stochastic model.

Typically, even when a scrubber functions properly, a small amount of $\mathrm{CO}_{2}$ will pass through the scrubber from the incoming path to the outgoing path. We define this phenomenon as $\mathrm{CO}_{2}$ channeling. Each $\mathrm{CO}_{2}$ channeling event happens randomly and is not considered as a fault. However, $\mathrm{CO}_{2}$ channeling will lead to the three $\mathrm{CO}_{2}$ related faults, and our stochastic model will reveal the mechanism.

Considering the randomness of $\mathrm{CO}_{2}$ channeling events, an arrival of $\mathrm{CO}_{2}$ at the outlet of a scrubber canister can be modeled as a Poisson process. We define a Poisson counter $N(t)$ such that $N(t)$ satisfies the following Poisson distribution:

$$
P[(N(t+\tau)-N(t))=d]=\frac{e^{-\lambda \tau}(\lambda \tau)^{d}}{d !}
$$

where $d=\{0,1, \cdots\}$ is the index of events and $\lambda$ is the expected number of events over unit length of time. Since each $\mathrm{CO}_{2}$ channeling event happens independently, we define $d N(t)$ as one single $\mathrm{CO}_{2}$ channeling event over $d t$.

The amount of the $\mathrm{CO}_{2}$ channeling during one event can be modeled by a scaling factor, and it is affected by two factors: the total volume of $\mathrm{CO}_{2}$ absorbed by the scrubber $y$ and the volume of $\mathrm{CO}_{2}$ coming into a scrubber canister $\mathscr{U}$. Then, the scaling factor $G$ can be described as a function of $y$ and $\mathscr{U}$ such that

$$
G=G(y(t), \mathscr{U}(t, n)) .
$$

Since we define $d N(t)$ as an event of $\mathrm{CO}_{2}$ channeling within $d t$, the change in $\mathrm{CO}_{2}$ volume in the outgoing path due to $\mathrm{CO}_{2}$ channelings can be expressed as

$$
d x=G(y(t), \mathscr{U}(t, n)) d N(t) .
$$

The two parameters for the Poisson process, $G$ and $\lambda$, need to be carefully selected to reflect the physical behavior of the rebreather. At the beginning of the rebreather use, $\mathrm{CO}_{2}$ channelings are very unlikely, but as the scrubber being consumed, the probability of $\mathrm{CO}_{2}$ channeling increases. After the scrubber is depleted, $\mathrm{CO}_{2}$ channeling will happen with probability 1 . According to this, $\lambda$ of the Poisson process can be assumed to be zero for a new scrubber but will become $\infty$ as the scrubber is getting fully consumed. Suppose the amount of $\mathrm{CO}_{2}$ absorbed by the scrubber $y$ is zero initially and its maximum capacity is $y_{\max }$, then the rate parameter should satisfy

$$
\begin{array}{ll}
\lambda(0)=0, & \lambda\left(y_{\max }\right)=\infty \\
\dot{\lambda}(0)=0, & \dot{\lambda}\left(y_{\max }\right)=0 .
\end{array}
$$

One possible function that satisfies the above conditions is

$$
\lambda(y)=\frac{y(t)}{y_{\max }-y(t)}
$$

where $y_{\max }$ is the maximum capacity of the $\mathrm{CO}_{2}$ scrubber and $y(t)$ is the remaining $\mathrm{CO}_{2}$ absorption capacity of the $\mathrm{CO}_{2}$ scrubber.

The function $G(y, \mathscr{U})$ will describe how much of $\mathrm{CO}_{2}$ could pass through a scrubber when a $\mathrm{CO}_{2}$ channeling event happens. Initial possible amount of $\mathrm{CO}_{2}$ channeling can be assumed to be zero for a new scrubber, but when a $\mathrm{CO}_{2}$ scrubber reaches its maximum capacity, all $\mathrm{CO}_{2}$ coming into the scrubber will pass through. Let us assume that $G$ is a polynomial function increasing from zero to $\mathscr{U}$ as $y(t)$ goes from zero to $y_{\max }$, then one possible choice of function $G$ is

$$
G(y, \mathscr{U})=\left(\frac{y(t)}{y_{\max }}\right)^{\alpha} \mathscr{U}(t, n)
$$

where $\alpha>0$ is a stretching factor. We see that when $y=0$, no $\mathrm{CO}_{2}$ channeling can happen, but when $y=y_{\max }$, all $\mathrm{CO}_{2}$ in the incoming path will pass through the scrubber in a single channeling event.

\section{D. $\mathrm{CO}_{2}$ Flow under $\mathrm{CO}_{2}$ related Faults}

In this subsection, we will specify $\mathrm{CO}_{2}$ flow dynamics in the rebreather induced by $\mathrm{CO}_{2}$ related faults using the stochastic $\mathrm{CO}_{2}$ channeling model with respect to $x$ and $y$. Parameters and variables of the model are summarized in table I.

By the assumption that gas in the incoming path does not flow to the outgoing path during inhalation, $\mathrm{CO}_{2}$ related 
TABLE I

VARIABLES AND PARAMETERS OF THE $\mathrm{CO}_{2}$ FLOW MODEL

\begin{tabular}{|c|c|c|}
\hline Parameter & Value/unit & Meaning \\
\hline$x$ & {$[L]$} & Volume of $\mathrm{CO}_{2}$ existing in outgoing path \\
$y$ & {$[L]$} & Volume of $\mathrm{CO}_{2}$ absorbed by $\mathrm{CO}_{2}$ scrubber \\
$p$ & {$[L]$} & Overall $\mathrm{CO}_{2}$ flow in rebreather \\
$\mathscr{U}$ & {$[L / \mathrm{min}]$} & $\mathrm{CO}_{2}$ exhalation rate \\
$v$ & {$[L / m i n]$} & Inhalation rate \\
$c_{1}$ & & Ratio of $\mathrm{CO}_{2}$ in the outgoing path \\
$c_{2}$ & & $\mathrm{CO}_{2}$ generation ratio \\
$s$ & & Heart rate ratio $\left(\mathrm{HR}^{2} / \mathrm{HR}_{\text {ref }}\right)$ \\
\hline
\end{tabular}

scrubber faults do not have influence on $\mathrm{CO}_{2}$ flow during inhalation. Thus, (5) and (6) still hold.

However, the faults affect $\mathrm{CO}_{2}$ flow during exhalation. The volume of $\mathrm{CO}_{2}$ in the outgoing path $x$ is as defined in (15). The volume of $\mathrm{CO}_{2}$ absorbed by the scrubber $y$ is determined by the difference between the volume of $\mathrm{CO}_{2}$ arriving at the inlet of a scrubber canister due to exhalation and the volume of a $\mathrm{CO}_{2}$ channeling event.

During exhalation $\left(t \in I_{2}\right)$ :

$$
\begin{aligned}
& d x=G(y(t), \mathscr{U}(t, n)) d N(t) \\
& d y=\mathscr{U}(t, n) d t-d x
\end{aligned}
$$

where $x(0)=0, y(0)=0$ and $I_{2}=\left[\frac{\left(n+\frac{1}{2}\right) T}{\chi(s)}, \frac{(n+1) T}{\chi(s)}\right)$ for $n=$ $\{0,1,2, \cdots\}$.

We define $p$ such that $p(t)=x(t)+y(t)$ which represents the total $\mathrm{CO}_{2}$ flow in the system, i.e., how much $\mathrm{CO}_{2}$ enters or leaves a rebreather. Then, the rebreather system can be modeled as a stochatic system with $p(t)$ and $x(t)$ as state variables.

During inhalation $\left(t \in I_{1}\right)$ :

$$
\begin{aligned}
& d x=-\frac{v(t)}{V_{L}} \cdot x(t) d t \\
& d p=d x
\end{aligned}
$$

During exhalation $\left(t \in I_{2}\right)$ :

$$
\begin{aligned}
d x & =G(p(t)-x(t), \mathscr{U}(t, n)) d N(t) \\
d p & =\mathscr{U}(t, n) d t
\end{aligned}
$$

where $x(0)=0, p(0)=0, \quad I_{1}=\left[\frac{n T}{\chi(s)}, \frac{\left(n+\frac{1}{2}\right) T}{\chi(s)}\right)$ and $I_{2}=$ $\left[\frac{\left(n+\frac{1}{2}\right) T}{\chi(s)}, \frac{(n+1) T}{\chi(s)}\right)$ for $n=\{0,1,2, \cdots\}$.

The volume of $\mathrm{CO}_{2}$ in the outgoing path $x$ will be measured by a $\mathrm{CO}_{2}$ sensor. However, the total $\mathrm{CO}_{2}$ flow $p$ is not able to be measured. Thus, the noisy measurement $m(t)$ for $x(t)$ can be modeled as an output of the stochastic systems in (21)-(24).

$$
m(t)=\left[\begin{array}{ll}
1 & 0
\end{array}\right]\left[\begin{array}{c}
x(t) \\
p(t)
\end{array}\right]+v(t)
$$

where $v(t)$ represents measurement noise.

\section{Particle Filter}

Since the model is driven by a Poisson process, there are limited choices of filters for state estimation. In particular, Kalman filter may not be very efficient. In this paper, the particle filter is applied to estimate the states $x(t)$ and $p(t)$. We first briefly introduce the particle filtering algorithm [18], and then apply the filter to estimate the states. The particle filter is performed by the iteration of the following four steps.

(i) Particle Creation

To initialize the filter, $N$ samples for each $x$ and $p$ are randomly generated from the initial probability distribution functions $\rho\left(x_{0}\right)$ and $\rho\left(p_{0}\right)$. These samples are denoted as $x_{i}^{+}(0)$ and $p_{i}^{+}(0)(i=1, \cdots, N)$, respectively. The values of the mean and the variance of the initial particles are chosen according to the fact that the volume of $\mathrm{CO}_{2}$ is always positive and the amount of $\mathrm{CO}_{2}$ is very small when an event of $\mathrm{CO}_{2}$ channeling occurs. The values we empirically found are on the order of $10^{-8}$. Since these values are very small, A scaling factor $\gamma$ is used to increase the value of particles to reduce numerical errors. The value used for simulation is $\gamma=10^{6}$

\section{(ii) Prediction (Diffusion)}

At each time step $k$, the particles are propagated to the next time step based on the system equations:

During inhalation $\left(k h \in I_{1}\right)$ :

$$
\begin{aligned}
& x_{i}^{-}((k+1) h)=x_{i}^{+}(k h)-\gamma c_{1}(l) v(k h) h \\
& p_{i}^{-}((k+1) h)=p_{i}^{-}(k h)+y_{i}^{-}((k+1) h)-y_{i}^{-}(k h)
\end{aligned}
$$

During exhalation $\left(k h \in I_{2}\right)$ :

$$
\begin{aligned}
& x_{i}^{-}((k+1) h)=x_{i}^{+}(k h)+G(k h) d N(t) \\
& p_{i}^{-}((k+1) h)=p_{i}^{+}(k h)+\mathscr{U}(k h, n) h
\end{aligned}
$$

where $G(k h)=G\left(p_{i}^{+}(k h)-y_{i}^{+}(k h), \mathscr{U}(k h, n)\right), i=\{1, \cdots, N\}$ and $h$ is the step size.

A Poisson jump $d N(t)$ is a continuous-time process, but it can be simulated in discrete-time as Poiss $(k h)$. Computational implementation of a Poisson process is introduced in [19], [20], [21]. In this paper, a Poisson process is simulated in the way suggested by [19]. and Poiss $(k h)$ represents the implementation of the Poisson counter during the time interval $(k h,(k+1) h)$ where $\mathrm{h}$ is a small step size. If a randomly generated number which is uniformly distributed in the interval $[0,1]$ is smaller than $1-e^{-h \lambda}$, then Poiss $(k h)=1$; otherwise, $\operatorname{Poiss}(k h)=1$. Then, we can express (28) as

$$
x_{i}^{-}((k+1) h)=x_{i}^{+}(k h)+G(k h) \operatorname{Poiss}(k h) .
$$

(iii) Likelihood Evaluation

We can obtain the output values using (25) as

$$
m(k h)=\left[\begin{array}{ll}
1 & 0
\end{array}\right]\left[\begin{array}{c}
x_{i}^{-}(k h) \\
p_{i}^{-}(k h)
\end{array}\right]+v(k h)
$$

where $v(k h) \sim \mathscr{N}(0, R)$.

After measuring $m(k h)$, the conditional relative likelihood is computed to evaluate $P\left(m(k h) \mid x_{i}^{-}(k h), p_{i}^{-}(k h)\right)$. Given that noise $v$ is Gaussian, for a specific measurement $m^{*}$, a relative likelihood $q_{i}$ can be computed as 


$$
\begin{aligned}
q_{i} & =P\left[\left(m_{k}=m^{*}\right) \mid\left(x_{k}=x_{i}^{-}(k h), p_{k}=p_{i}^{-}(k h)\right)\right] \\
& =P\left[v_{k}=m^{*}-h\left(x_{i}^{-}(k h), p_{i}^{-}(k h)\right)\right] \\
& \sim \frac{\exp \left(\frac{-r_{i}^{T}(k h) R^{-1} r_{i}(k h)}{2}\right)}{(2 \pi)^{n / 2}|R|^{1 / 2}}
\end{aligned}
$$

where $r_{i}(k h)=\left[m^{*}-h\left(x_{i}^{-}(k h), p_{i}^{-}(k h)\right)\right]$ and $n=1$. Then, in order to ensure that the sum of all the likelihoods is equal to one, the relative likelihoods are normalized as $\bar{q}_{i}=q_{i} / \sum_{j=1}^{N} q_{j}$.

(iv) Resampling

Particles are resampled by using the following two steps for $i=1, \cdots, N$.

1) Generate a uniformly distributed random number $r$ on $[0,1]$.

2) If $\sum_{m=1}^{j-1} \bar{q}_{m}<r$ but $\sum_{m=1}^{j} \bar{q}_{m} \geq r$, then $x_{i}^{+}(k h)=x_{j}^{-}(k h)$ and $p_{i}^{+}(k h)=p_{j}^{-}(k h)$.

Then, the estimates of $x$ and $p$ at time $k h$ are determined by the statistical mean of the updated particles such that

$$
\left[\begin{array}{c}
\tilde{x}(k h) \\
\tilde{p}(k h)
\end{array}\right]=\left[\begin{array}{c}
\frac{1}{N} \sum_{i=1}^{N}\left\{x_{i}^{+}(k h)\right\} \\
\frac{1}{N} \sum_{i=1}^{N}\left\{p_{i}^{+}(k h)\right\}
\end{array}\right] .
$$

\section{Fault Diagnosis and PRognosis}

Our $\mathrm{CO}_{2}$ flow model helps the detection of the $\mathrm{CO}_{2}$ related scrubber faults. Fault diagnosis and prognosis are achieved using the particle filtering results based on the $\mathrm{CO}_{2}$ flow model.

\section{A. Fault Detection and Isolation for Rebreathers}

1) $\mathrm{CO}_{2}$ bypass: The estimate of the volume of $\mathrm{CO}_{2}$ channeling over one single period of exhalation $\Delta \tilde{x}$ is obtained using $\tilde{x}$ such that

$$
\Delta \tilde{x}(n)=\tilde{x}((n+1) T)-\tilde{x}\left(\left(n+\frac{1}{2}\right) T\right),
$$

and the occurrence of $\mathrm{CO}_{2}$ bypass can be determined by summating the volume of $\mathrm{CO}_{2}$ channelings over a finite time window such that the decision of $\mathrm{CO}_{2}$ bypass is made based on the following criterion:

$$
\sum_{i=n-M+1}^{n} \Delta \tilde{x}(i)>H_{1}
$$

where $M$ is the width of the time window, and $H_{1}$ is the threshold for detection.

Equation (35) itself is enough to detect $\mathrm{CO}_{2}$ bypass. However, a more detrimental factor induced by $\mathrm{CO}_{2}$ bypass fault is the partial pressure of $\mathrm{CO}_{2}, \mathrm{ppCO}_{2}$. We assume that the pressure inside the rebreather is the same as the ambient pressure. Provided that the ambient pressure $P_{a m b}$ and the volume of gas in the outgoing path $V_{L}$ are known, $\mathrm{ppCO}_{2}$ can be computed similarly to $\mathrm{ppO}_{2}$ in [10] as

$$
\operatorname{ppCO}_{2}=100 \cdot c_{1} \cdot P_{a m b}=\frac{\tilde{x} \cdot P_{a m b}}{V_{L}}
$$

Then, with a threshold value $\mathrm{H}_{2}$, we can detect $\mathrm{CO}_{2}$ bypass by

$$
\mathrm{ppCO}_{2}>\mathrm{H}_{2} \text {. }
$$

The fault of $\mathrm{CO}_{2}$ bypass will be detected if either of the two thresholds, $\mathrm{H}_{1}$ or $\mathrm{H}_{2}$, are exceeded.

2) $\mathrm{CO}_{2}$ scrubber exhaustion and $\mathrm{CO}_{2}$ scrubber breakthrough: $\mathrm{CO}_{2}$ scrubber exhaustion and $\mathrm{CO}_{2}$ scrubber breakthrough happen when $\mathrm{CO}_{2}$ absorption capability is lost. To detect these faults we need to compare $\Delta \tilde{x}$ with the volume of $\mathrm{CO}_{2}$ exhaled over one breathing period. Let us define

$$
\Delta \mathscr{U}(n)=\int_{\left(n+\frac{1}{2}\right) T}^{(n+1) T} \mathscr{U}(\tau, n) d \tau
$$

where $n=\{0,1,2, \cdots\}$.

We can detect scrubber exhaustion or breakthrough by first checking whether

$$
\Delta \mathscr{U}(n)-\Delta \tilde{x}(n)<H_{3}
$$

where $H_{3}$ is a threshold value. If the threshold is exceeded, then complete $\mathrm{CO}_{2}$ channeling events is happening. Using $\tilde{x}$ and $\tilde{p}$ obtained by (33), we can determine whether the fault will lead to scrubber exhaustion or scrubber breakthrough. If the scrubber does not reach its maximum absorption capacity yet, complete $\mathrm{CO}_{2}$ channelings indicate scrubber breakthrough. Let us define the remaining scrubber capacity $(\mathscr{C})$ as

$$
\mathscr{C}=1-\frac{y(t)}{y_{\max }}=1-\frac{p(t)-x(t)}{y_{\max }}
$$

where $x$ is the volume of $\mathrm{CO}_{2}$ in the outgoing path, $y$ is the current $\mathrm{CO}_{2}$ absorption capacity of the scrubber, $p$ is the overall $\mathrm{CO}_{2}$ flow and $y_{\max }$ is the maximum absorption capacity of the srubber. At the time of the fault occurrence, if

$$
\tilde{\mathscr{C}}<H_{4}
$$

where $H_{4}$ is a threshold on the scrubber depletion, then the fault is identified as scrubber exhaustion, otherwise scrubber breakthrough.

\section{B. Prognostics and Health Management}

We can use the state estimates from the Particle Filter to predict future $\mathrm{CO}_{2}$ channeling occurrences by propagating the state equations of the rebreather $\mathrm{CO}_{2}$ flow model forward in time. Prognosis for the faults are implemented according to the fault criteria in (35), (37), (39) and (41) using the predicted values $\hat{x}$ and $\hat{p}$ obtained by Algorithm 1 instead of estimated values $\tilde{x}$ and $\tilde{p}$. The fault prediction algorithm at $t=k h$ for the future states at $t=(k+l) h$ is as follows. Note that the tilde represents the state estimate and the hat represents the state prediction. 


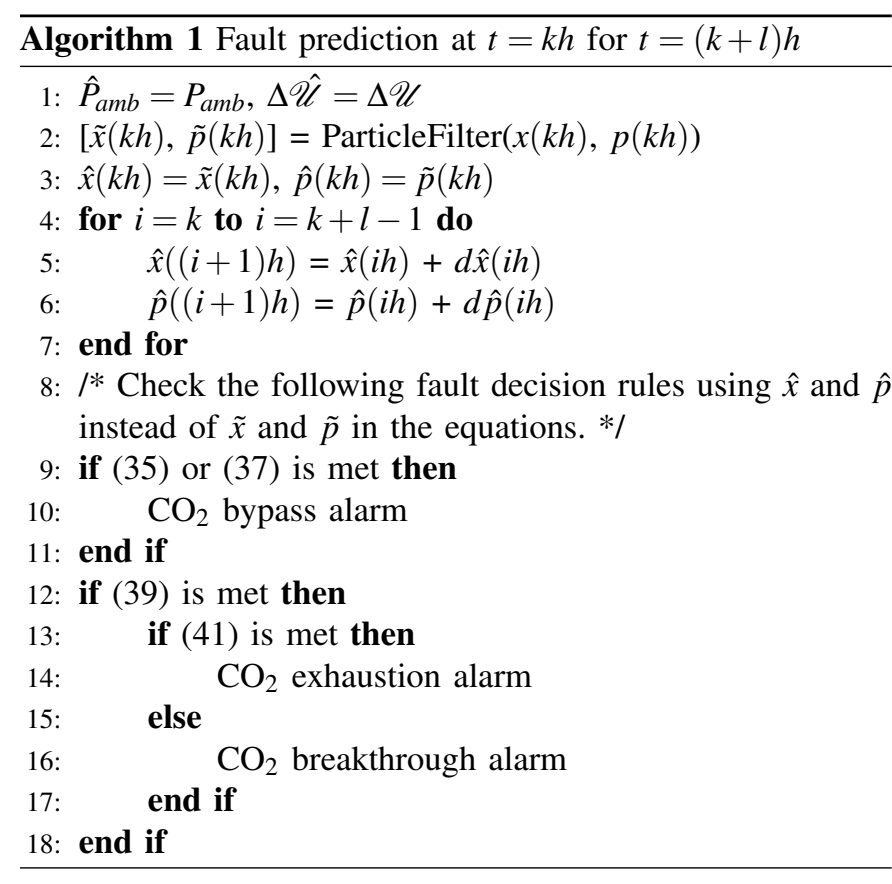

\section{Simulation Results}

We perform simulations of the rebreather based on our stochastic $\mathrm{CO}_{2}$ flow model at a constant depth with ambient pressure as $P_{a m b}=1$ to demonstrate the $\mathrm{CO}_{2}$ related rebreather faults. The parameters we used in simulations are: the number of particles as $N=800$; the capacity of the scrubber as $y_{\max }=10$; the size of the outgoing path in the breathing loop as $V_{L}=30$; the heart rate ratio as $s=1$; the stretching factor for the function $G$ as $\alpha=10^{-6}$; the window size for the detection of the $\mathrm{CO}_{2}$ bypass fault as $M=1$; and the time step as $h=0.05$.
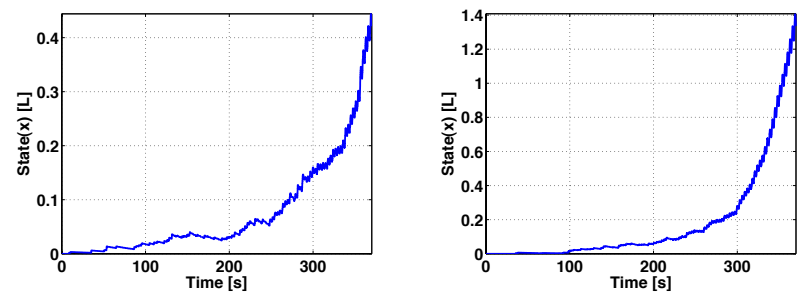

(a) State $x$ under scrubber exhaus- (b) State $x$ under scrubber breaktion. through.

Fig. 3. The amount of $\mathrm{CO}_{2}$ in the outgoing path under scrubber exhaustion and scrubber breakthrough up to $t=370$ are shown in (a) and (b), respectively. Their patterns are almost identical except that they happen at different times. Note the difference in values of the state $x$ at time $t$ between the cases of scrubber exhaustion and scrubber breakthrough. $\mathrm{CO}_{2}$ bypass happens due to the accumulated $\mathrm{CO}_{2}$ in the outgoing path before the exhaustion or the breakthrough.

The $\mathrm{CO}_{2}$ flow patterns under the faults of scrubber exhaustion and scrubber breakthrough are described in Fig. 3. Scrubber exhaustion happens when the scrubber is depleted and Fig. 3(a) shows that there is a dramatic increase in $\mathrm{CO}_{2}$ in the outgoing path after $t=330$ under the scrubber exhaustion fault. The scrubber breakthrough is intentionally made such that the maximum capacity of the scrubber $y_{\max }$ under scrubber breakthrough is reduced to $0.85 \cdot y_{\max }$ from the beginning of the simulation. The $\mathrm{CO}_{2}$ flow pattern under scrubber breakthrough is similar to the scrubber exhaustion case, but when scrubber breakthrough occurs, the increase in $\mathrm{CO}_{2}$ in the outgoing path comes earlier as in Fig. 3(b), so there exists more $\mathrm{CO}_{2}$ in the outgoing path in the same amount of dive time after $\mathrm{CO}_{2}$ breakthrough occurs. $\mathrm{CO}_{2}$ bypass happens at the early stages of scrubber exhaustion and scrubber breakthrough as $\mathrm{CO}_{2}$ is getting accumulated in the outgoing path due to $\mathrm{CO}_{2}$ channeling events.

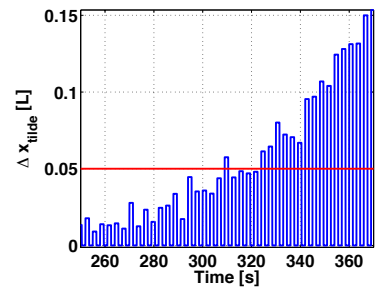

(a) $\Delta \tilde{x}$ with the threshold value as (b) Estimated $\mathrm{ppCO}_{2}$ with the $H_{1}=0.05$

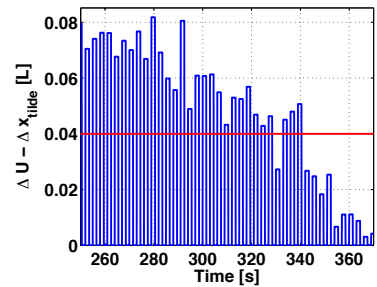

(c) $\Delta U-\Delta \tilde{x}$ with the threshold value (d) The remaining capacity of the as $H_{3}=0.04$.

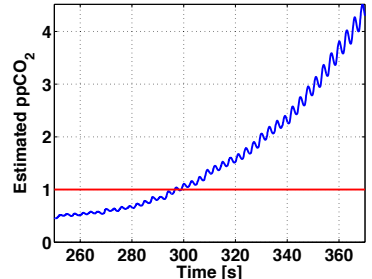

threshold value as $H_{2}=1$

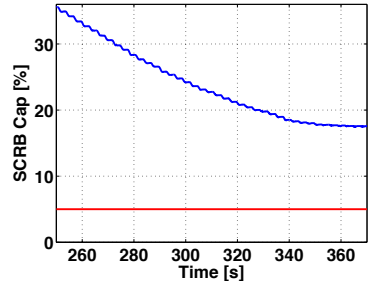

scrubber with the threshold value as $H_{4}=0.05$.
Fig. 4. Parameters for fault diagnosis are introduced with the threshold values (red lines) in the case of scrubber breakthrough. For $\mathrm{CO}_{2}$ bypass fault detection, $\Delta \tilde{x}$ and $\mathrm{ppCO}_{2}$ are used as in (a) and (b). Parameters for scrubber exhaustion/breakthrough detection are presented in (c) and (d).

To demonstrate the $\mathrm{CO}_{2}$ related fault diagnosis and prognosis algorithms, we implement the particle filter on our stochastic $\mathrm{CO}_{2}$ flow model. An example of fault diagnosis for scrubber breakthrough is presented in Fig. 4. The detection criteria on $\mathrm{CO}_{2}$ bypass fault are shown with threshold values in Figs. 4(a) and 4(b). The occurrence of $\mathrm{CO}_{2}$ bypass will be determined by checking $\Delta \tilde{x}$ and estimated $\mathrm{ppCO}_{2}$. The criteria on the scrubber exhaustion/breakthrough are presented in Figs. 4(c) and 4(d). By setting a threshold value for $\Delta U-\Delta \tilde{x}$ as 0.04 , scrubber exhaustion/breakthrough can be detected, and the remaining capacity of the scrubber can be checked to determine which fault between scrubber exhaustion and scrubber breakthrough happens.

The system propagation for fault prognosis is shown in Fig. 5. We use the same criteria and threshold values as used in previous figures. We see that the particle filter estimates are 
updated before $t=280$, and system propagation is performed as the red line segments in Fig. 5. Then, a prediction for scrubber exhaustion/breakthrough can be achieved using $\Delta U-\Delta \tilde{x}$ in Fig. 5(a) and the remaining capacity of the scrubber as $100 \cdot \mathscr{C}$ in Fig. 5(b). Compared with the simulation results of fault diagnosis in Figs. 4, our model is optimistic, e.g., the predicted time of the fault occurrence is about 30 seconds later than the actual fault occurrence time. To improve the accuracy of the fault prediction under scrubber breakthrough, the change in the actual maximum capacity of a $\mathrm{CO}_{2}$ scrubber needs to be studied as well.
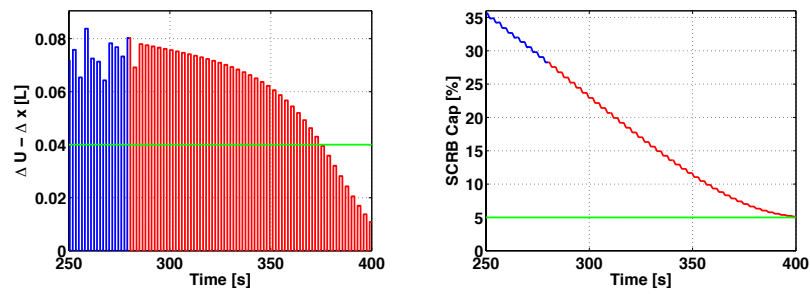

(a) $\Delta U-\Delta \tilde{x}$ with the threshold value (b) The remaining capacity of the as $H_{3}=0.04$. scrubber with the threshold value as $H_{4}=0.05$.

Fig. 5. State propagation after $t=280$ for a scrubber breakthrough prediction. The blue and the red curves indicate the particle filter estimate and the system propagation, respectively. The threshold values are introduced as green lines.

\section{CONCLUSIONS}

We have presented fault diagnosis and prognosis algorithms for three rebreather faults: $\mathrm{CO}_{2}$ bypass, scrubber exhaustion and scrubber breakthrough. As part of our achievements, we have investigated the process of $\mathrm{CO}_{2}$ flow inside a rebreather and the influence of the faults on $\mathrm{CO}_{2}$ flow. Consequently, we have developed a stochastic model of a $\mathrm{CO}_{2}$ channeling event whose cumulative occurences result in the rebreather faults and a $\mathrm{CO}_{2}$ flow model using the stochastic model. We have shown that the particle filter is applicable to obtain the state estimates of $\mathrm{CO}_{2}$ flow, and the fault diagnosis/prognosis algorithms are tested in simulations based on the particle filter estimates.

\section{ACKNOWLEDGMENT}

The research work is supported by ONR grants N0001408-1-1007, N00014-09-1-1074, and N00014-10-10712 (YIP), and NSF grants ECCS-0841195 (CAREER), CNS-0931576, and ECCS-1056253, and GTRI

\section{REFERENCES}

[1] R. Klos, "Principles of work of different types of underwater breathing apparatus," Polish Maritime Research, vol. 15, no. 4, pp. 72-84, 2008.

[2] C. J. Lambertsen, "Carbon dioxide tolerance and toxicity," Environmental Biomedical Stress Data Center, Institute for Environmental Medicine, University of Pennsylvania Medical Center, Tech. Rep., 1971.

[3] R. D. Vann, N. W. Pollock, and P. J. Denoble, "Rebreather fatality investigation." American Academy of Underwater Sciences (AAUS), 2007.

[4] “Deep Life, Ltd.” [Online]. Available: http://deeplife.co.uk
[5] J. R. Clarke, "Computer modeling of the kinetics of $\mathrm{CO}_{2}$ absorption in rebreather scrubber canisters," vol. 3. Proceedings of OCEANS, 2001, pp. 1738-1744.

[6] S. Tetlow and S. Jenkins, "The use of fault tree analysis to visualise the importance of human factors for safe diving with closed-circuit rebreathers (CCR)," International Journal of the Society for Underwater Technology, vol. 26, no. 3, pp. 105-113, 2005

[7] P. G. Sexton and M. L. Nuckols, "Computer simulation of breathing systems for divers," Journal of Engineering for Industry - Transactions of the ASME, vol. 105, pp. 54-59, 1983.

[8] T. A. Antoon and M. L. Nuckols, "Simulation of a conceptual closedcircuit underwater breathing apparatus," Current Practices and New Technology in Ocean Engineering, vol. 12, pp. 79-86, 1987.

[9] A. Baz and J. Gilheany, "Modeling the dynamics of breathing circuits for underwater divers," Journal of Fluid Control, vol. 19, pp. 58-77, 1988.

[10] F. Garofalo, S. Manfredi, and S. Santini, "Modelling and control of oxygen partial pressure in an underwater breathing apparatus with gas recycle," in European Control Conference, 2003, pp. 927-932.

[11] D. Veinot, A. MacLean, and C. MacGregor, "Chemical and physical factors affecting the absorption capability of calcium hydroxide based carbon dioxide absorbents," in The characterization of carbon dioxide absorbing agents for life support equipment : presented at the Winter Annual Meeting of the American Society of Mechanical Engineers, vol. 10, 1982, pp. 47-56.

[12] C. D. MacGregor and M. G. Fraser, "Effect of pressure on the efficiency of carbon dioxide absorbents," in The characterization of carbon dioxide absorbing agents for life support equipment : presented at the Winter Annual Meeting of the American Society of Mechanical Engineers, vol. 10, 1982, pp. 75-82.

[13] E. Buban and J. Haughey, "Lithium hydroxide as a $\mathrm{CO} 2$ scrubber in closed circuit breathing apparatus," in The characterization of carbon dioxide absorbing agents for life support equipment : presented at the Winter Annual Meeting of the American Society of Mechanical Engineers, vol. 10, 1982, pp. 151-155.

[14] S. P. Tomlinson, D. G. Tilley, and C. R. Burrows, "Computer simulation of the human breathing process," IEEE Engineering in Medicine and Biology Magazine, vol. 13, no. 1, pp. 115-124, 1994.

[15] S. P. Tomlinson, J. K. W. Lo, and D. G. Tilley, "Computer simulation of human interaction with underwater breathing equipment," Proceedings of the Institution of Mechanical Engineers, Part H (Journal of Engineering in Medicine), vol. 208, no. H4, pp. 249-261, 1994.

[16] C. E. de Normalisation, "Respiratory equipment - self-contained rebreathing diving apparatus (EN14143:2003)," European Standard, 2003.

[17] D. Shier, J. Butler, and J. W. Hole, Hole's Human Anatomy \& Physiology. McGraw-Hill, 2002.

[18] D. Simon, Optimal State Estimation: Kalman, $H_{\infty}$, and Nonlinear Approaches. Wiley, 2006.

[19] R. W. Brockett, "Stochastic control," Lecture Notes, preprint.

[20] K. Burnecki, W. Hardle, and R. Weron, "Simulation of risk processes," in Encyclopedia of Actuarial Science. John Wiley \& Sons, 2006.

[21] L. Gustafsson, "Poisson simulation - a method for generating stochastic variations in continuous system simulation," Simulation, vol. 74, no. 5, pp. 264-274, 2000 . 